\title{
CREENCIAS ASOCIADAS A LA PRUEBA SIMCE DE EDUCACIÓN FÍSICA POR PARTE DE LOS DOCENTES DE EDUCACIÓN FÍSICA DE COLEGIOS PARTICULARES, SUBVENCIONADOS Y MUNICIPALES DE LA COMUNA DE SAN FELIPE BELIEFS ASSOCIATED WITH PROOF OF PHYSICAL EDUCATION SIMCE BY PHYSICAL EDUCATION TEACHERS COLLEGE SPECIFIC, SUPPORTED AND MUNICIPAL BOROUGH OF SAN FELIPE
}

\author{
Cajas Luna, Boris1; Barraza Gómez, Fernando1,2; Olivares Castro, Nataly1; Silva Pulgar,Teresa1; Villalobos Gaete, Doris1.
}

1 Escuela de Educación Física, Universidad Viña de Mar.

2 Laboratorio Motricidad Humana. Escuela de Educación Física, Pontificia Universidad Católica de Valparaíso.

CAJAS L.B.; BARRAZA G.F.; OLIVARES C.N.; SILVA P.T.; VILLALOBOS G.D. Creencias asociadas a la prueba SIMCE de educación física por parte de los docentes de educación física de colegios particulares, subvencionados y municipales de la comuna de San Felipe. Mot. Hum., 12(2): 21-25, 2011.

\section{RESUMEN}

Esta investigación de tipo caso descriptiva presenta como objetivo general conocer las creencias asociadas a la prueba SIMCE de educación física por parte de docentes de educación física de colegios particulares, subvencionados y municipales de la Comuna de San Felipe. Se llevo a cabo en el período Marzo-Diciembre del año 2011. Para ello se utilizaron técnicas cualitativas; entrevista en profundidad o no estructurada con preguntas abiertas para los profesores de educación física, con el fin de conocer las creencias asociadas a la prueba Simce de Educación Física. Expone contenidos relacionados con el sistema de medición educacional en Chile, dependencias administrativas, índices de sedentarismo, obesidad y las implicancias, de éstas, según estudios en el desempeño escolar y también en el proceso de enseñanzaaprendizaje. Se espera que esta investigación, otorgue información básica y detallada que permita fundamentar estudios posteriores de mayor profundidad y análisis, como también ser la base de futuros proyectos de acción en el campo del contexto educacional específicamente en el área de políticas de evaluación educacional en el país.

Palabras clave: Simce, Docentes, Capacidades Físicas, Dependencias Administrativas.

\section{INTRODUCCIÓN}

Desde la década del 60 se ha evaluado el resultado de los aprendizajes en el sistema educativo en Chile. El primer sistema instaurado se conoció como "Programa de Evaluación del Rendimiento Escolar" (PER) en 1982, su propósito principal fue contribuir a mejorar la calidad educativa a través de la descentralización de las responsabilidades y entregar una señal explícita al sistema educacional acerca de los objetivos de aprendizaje considerados como fundamentales por el Ministerio de Educación.(1) Posteriormente al descentralizarse el sistema educativo se instaló una nueva técnica de medición, llamada "Sistema de Medición de la Calidad de la Educación" (SIMCE) en 1988. (2)

El SIMCE es el Sistema Nacional de Evaluación de resultados de aprendizaje del Ministerio de Educación de Chile, que tiene como propósito principal contribuir al mejoramiento de la calidad y equidad de la educación. Este sistema permite conocer el desempeño de los estudiantes en diferentes áreas del ámbito cognitivo del Currículum Nacional, de acuerdo al contexto escolar y social en el que se desenvuelven.

Los resultados obtenidos en el año 1999, oportunidad en la que el SIMCE evaluó el mismo nivel de enseñanza, con los del año 2002, se advierte que en términos de resultados escolares no hubo avances significativos.

Los análisis al respecto dan cuenta de la presencia e influencia que ejercen dos conjuntos de variables: de una parte, aquellas relacionadas con el nivel socio-económico cultural de pertenencia de los estudiantes y por otra, las características de las escuelas a las que éstos estudiantes asisten. Pero existe otro conjunto de variables que pueden ser consideradas directamente modificables por cada escuela; se trata de variables 'intra-escuela', variables que requieren ser identificadas con el objetivo de potenciar su aporte como 'valor agregado' como son las prácticas pedagógicas y más específicamente, a las prácticas de enseñanza que llevan a cabo los docentes en las aulas de las escuelas del país. 
CAJAS L.B.; BARRAZA G.F.; OLIVARES C.N.; SILVA P.T.; VILLALOBOS G.D. Creencias asociadas a la prueba SIMCE de educación física por parte de los docentes de educación física de colegios particulares, subvencionados y municipales de la comuna de San Felipe. Mot. Hum., 12(2): 21-25, 2011.

Unir los resultados en el tiempo obliga a mirar el desarrollo integral de los niños y niñas del país, considerando no sólo lo cognitivo, sino que también lo emocional y físico, factores que afectan directamente los resultados intelectuales.

En Chile, entre los años 2003 - 2010 se realizaron dos encuestas nacionales de salud, arrojando un aumento significativo de la obesidad en un $25,1 \%$ de la población, para lo cual se busca implementar nuevas formas para bajar estos índices, por eso es que se extiende el sistema de evaluación SIMCE al ámbito físico y a través del Ministerio de Educación y la Subsecretaria de Deportes, se crea una aplicación piloto del SIMCE al subsector de educación física, planteando como objetivo diagnosticar la Condición Física de los estudiantes, y el impacto de esta condición en su calidad de vida. (3)

Sin embargo, todo sistema de evaluación debe considerar factores sociales como prejuicios, creencias y percepciones de la batería de test aplicada, en especial en el ámbito educativo, ya que el objetivo prioritario del SIMCE es mejorar la calidad de la educación.

Si se toma en cuenta que las creencias de los profesionales de la educación con respecto a las concepciones del SIMCE de educación física piloto, afectan el objetivo central del sistema de evaluación, se hace necesario conocer las creencias de los profesores, ya que son ellos quienes deberán generar los cambios para mejorar el sistema educacional.

Las creencias de la realidad determinan comportamientos de las personas frente a ella, como en el caso de los profesores quienes crean pensamientos, sentimientos, actitudes, expectativas y valoraciones, lo que influirá en su comportamiento frente al SIMCE y las estrategias utilizadas para mejorar "la calidad v/s resultado de evaluación.

Dada la falta de antecedentes sobre el tema en Chile, esta investigación se plantea como un estudio exploratorio. Su objetivo es lograr una primera aproximación descriptiva del fenómeno que interesa a partir de las diferentes dependencias administrativas en educación de la ciudad de San Felipe.

Existen varios factores implicados a la hora de determinar el nivel de la condición física de los alumnos, entre estos destacamos; factores genéticos, factores hereditarios, hábitos deportivos, hábitos alimenticios y tiempo de ocio. Actualmente, el ocio está directamente relacionado con la globalización, ya que afecta directamente a la función de la educación física, teniendo relación con el tiempo libre y de qué manera lo utilizan los alumnos, ya que hoy en día el uso de los videos juegos, las computadoras e Internet es de fácil acceso, siendo este un factor por el cual los alumnos dejan de lado las actividades lúdicas y deportivas que involucran un gasto energético.

Este estudio enfoca el SIMCE de educación física desde las creencias de los profesores de educación física, ya que es una forma directa de poder detectar los agentes que se ven involucrados a la hora de evaluar la condición física de los alumnos. Otro factor importante es conocer las creencias que los mismos docentes plantean acerca de los resultados arrojados por el SIMCE de educación física y si existe congruencia entre los discursos de los profesores de esta área sobre la elección y aplicación de las pruebas, y los resultados que arrojaron estas.

\section{MATERIAL Y METODOS}

Corresponde a un estudio cualitativo, de tipo caso único, de caso porque es un método de aprendizaje acerca de una situación compleja; está basado en el entendimiento comprensivo de una situación, el cual se puede obtener a través de la descripción y análisis de la situación, se toma como un conjunto y dentro de su contexto. De caso único, porque es un sistema acotado, es un objeto más que un proceso. Los casos que son de interés en educación son las personas y los programas. Se estudia tanto lo que tiene de único como lo que tiene en común. Nos interesa aprender como funciona en sus afanes (4).

$\mathrm{Su}$ diseño es no experimental, por consiguiente, no se manipulan deliberadamente los atributos, no obstante se busca principalmente observar la realidad tal cual es, del modo en que se manifiesta habitualmente. Todo esto a través del discurso que utilizan los profesores de educación física participantes en la investigación.

Principalmente esta investigación está basada en observar diversos aspectos subjetivos difíciles de medir objetivamente. Su principal objetivo es lograr una descripción e interpretación holística del asunto.

Este estudio contempla los atributos; Simce de Educación Física, Profesor de Educación Física, Dependencias Administrativas y Creencias. De los atributos mencionados, y luego del análisis de discurso, surgen las categorías de educación física, dependencias administrativas, Simce de Educación Física, Profesor, Resultados Simce de Educación Física.

Con referente a la población utilizada para este estudio, estuvo compuesta por 8 profesores de educación física 
CAJAS L.B.; BARRAZA G.F.; OLIVARES C.N.; SILVA P.T.; VILLALOBOS G.D. Creencias asociadas a la prueba SIMCE de educación física por parte de los docentes de educación física de colegios particulares, subvencionados y municipales de la comuna de San Felipe. Mot. Hum., 12(2): 21-25, 2011.

de establecimientos municipales, subvencionados y particulares de la comuna de San Felipe.

La muestra es no probabilística e intencionada. En tanto, la técnica utilizada para la recolección de datos fueron entrevistas en profundidad, entendiéndolas como la obtención de información sobre un determinado contenido y a partir de éste se establece una lista de temas, en relación a los focos de la entrevista, quedando ésta a libre discreción del entrevistador, quien puede sondear razones y motivos, ayudar a establecer determinado factor, pero sin ajustarse a una estructura formalizada de antemano (5).

\section{RESULTADOS}

Los profesores expresan su opinión acerca del poco tiempo destinado para las clases de educación física e indican que este es un factor que influye de manera significativa en los objetivos y resultados del SIMCE de educación física. Señalan además, la posibilidad u opción de aumentar el número de horas semanales de la actividad deportiva, con la idea de combatir los niveles de sedentarismo y obesidad.

Se identifica así, que no se forma a los alumnos de manera íntegra y armoniosa, ya que la clase de educación física se realiza una vez a la semana con un tiempo de 90 min. Siendo que la OMS estipula que lo recomendado para la realización de actividad física es de tres veces por semana con un tiempo mínimo de 60 minutos por sesión.

Cabe señalar entonces, que debido a la cantidad de tiempo en las clases de educación física es imposible poder formar y evaluar a los alumnos de manera individual tomando en cuenta las capacidades que posee cada uno, además existe una incoherencia con respecto a las recomendaciones que plantean los estamentos mundiales de la salud, refiriéndose a aportar en la prevención de las enfermedades transmisibles que afectan en un gran porcentaje a la sociedad. Hay un desconocimiento sobre el estado de la salud de los alumnos, en algunas dependencias administrativas no se exige un examen médico que certifique que el alumno está en condiciones de realizar actividad física, por lo tanto, si un niño presentara alguna patología, el SIMCE de educación física no toma en cuenta estos factores, solo mide la condición física de los alumnos y no se sabe si el niño puede llegar a presentar alguna complicación a la hora de realizar las clases normales o más aún en el momento de ejecutar la prueba. Entonces como solución, los establecimientos y los mismos profesores de educación física debiesen exigir los documentos que sean necesarios para conocer el estado de salud de los alumnos, que les permitan medir y realizar los trabajos óptimos a las exigencias que requiera un niño con condiciones de salud especiales.

Por lo tanto, es trascendente señalar que la importancia y el respeto que entregue el profesor de educación física y el establecimiento educacional a la clase de educación física, es relevante para concretar los objetivos que ésta requiere, desde la formación académica, moral y corporal para el desarrollo óptimo del alumno. De tal manera que depende del profesor el buen uso que le dé a su clase y cómo optimiza los tiempos necesarios para lograr los objetivos de ésta. No obstante a los profesores de educación física les ocupan las horas para otras actividades, les ocupan el patio que es su sala de clases y es la asignatura que debe estar disponible para la realización de otras actividades sujetas a modificaciones del horario.

Uno de los factores influyentes en la creación del SIMCE de educación física son el aumento de los niveles de obesidad y el sedentarismo, por lo tanto, se afirman las creencias que exponen los profesores de educación física sobre los motivos determinantes de ésta nueva medición, acerca de implementar nuevas medidas para combatir estos problemas de salud que afligen al país. Pero, se cuestionan los docentes porque no se implementó esta medición años atrás, cuando los niveles de sedentarismo y obesidad venían en aumento. No obstante si analizamos los datos del año 2003 (6), ya existían niveles de sedentarismo y no se aplicaba ninguna prueba SIMCE, de lo cual se podría concluir que se está atacando la epidemia y no la prevención.

Los docentes señalan además, que si el objetivo es diagnosticar la condición física de los alumnos, los test usados para el logro de este fin no son los más apropiados, ya que miden a todos los alumnos por igual sin tomar en cuenta distintos factores influyentes en la condición física de éstos, como enfermedades no transmisibles, nivel de actividad física, factores psicológicos y sociales.

Las pruebas que se aplicaron en el SIMCE de educación física no fueron las que normalmente se utilizan en las clases de educación física, por ejemplo, los abdominales no son los que se trabajan con los alumnos, ya que tienen un ritmo forzado que obviamente al no tener un conocimiento de la ejecución, no se obtendrán buenos resultados. También el test de navette, es una medición que se había dejado de utilizar hace varios años y no se estaba utilizando en los colegios, entonces los alumnos no tenían conocimiento del ritmo de ejecución de la prueba.

La aplicación del SIMCE de educación física es aprobada por los profesores de educación física, sin embargo, 
plantean que es necesario realizar modificaciones tomando en cuenta los resultados de la aplicación piloto realizada. Estas modificaciones están referidas al cambio de algunas de las pruebas que se aplicaron y que se utilicen los resultados como instrumento para la creación de estrategias para combatir la obesidad y el sedentarismo.

La información entregada respecto a los resultados del SIMCE de educación física, fue solo por medios de comunicación, y no llegó información oficial a cada establecimiento para comunicar el nivel en que se encontraban los alumnos que rindieron esta prueba piloto del SIMCE de educación física, y así de este modo poder cumplir con lo planteado en un inicio por el Ministerio de Educación, en informar y orientar a los apoderados respecto al rendimiento de sus alumnos, clasificando a los colegios dependiendo de su rendimiento, tomando de este modo menos importancia al SIMCE de educación física, con respecto a los otros SIMCE.

\section{DISCUSIÓN}

La clase de educación física carece de mayor tiempo para el logro de la formación integral del alumno, cada uno de los profesores concuerda que a los educandos se les debe evaluar de acuerdo a sus necesidades, que no se toman en cuenta y que se pasan por alto, ya que no todos los establecimientos exigen un examen médico a los alumnos, por lo tanto el profesor al tener poco tiempo efectivo de clases y no contar con el conocimiento de las patologías de los alumnos, toma una responsabilidad extra al realizar y planificar su clase. Entonces a través del SIMCE se pide calidad en la educación, pero existen falencias con relación al tema de las horas de clases, además que a la mayoría de los profesores se les intervienen las clases, ya sea por actos, premiaciones, celebraciones, etc. Entonces estamos hablando de tres factores influyentes en la clase de educación física, el primero se enfoca al poco tiempo en cuanto horario de educación física, el segundo alude a las necesidades especiales de los niños y la falta de información médica, tercero y por último, la poca importancia y respeto la clase de educación física, como una asignatura de formación integral al igual que todas las demás.

Los profesores de educación física comenzaron a practicar con sus alumnos para la aplicación del SIMCE, sin embargo, al momento de la evaluación se encontraron con una gran sorpresa, se aplicaban pruebas que los alumnos desconocían; test con un ritmo de ejecución forzada, pruebas que excluían a los alumnos y que hacían que estos se exigieran más de lo que podían rendir, contradiciendo entonces con el objetivo general del SIMCE de educación física en medir la calidad y la condición física de los alumnos. Si todos los docentes de Chile se rigen por los planes y programas del ministerio, el SIMCE debiese haber creado pruebas en base a lo que el ministerio postula y a lo que se trabaja en los colegios, como por ejemplo: abdominales conocidos, pruebas que midan la capacidad aeróbica usados actualmente, ya que el test de Course Navette debido a su carácter contraproducente se descontinuó su uso en el sistema, por causa de que llevaba a una sobre exigencia al ejecutar la prueba por parte de los alumnos, obteniendo hace algunos años problemas graves en los alumnos durante la ejecución de la prueba.

Después de la aplicación del SIMCE de Educación Física quedó la expectativa acerca de los resultados que se obtuvieron y la incertidumbre de cómo los colegios habían sido evaluados. Estos resultados indicaron que la mayoría de los alumnos no posee una condición física satisfactoria, es por esto que en los colegios comenzó la preocupación de los profesores de educación física sobre preparar y mejorar la condición física de los alumnos, planificando en función de implementar posibles soluciones y en un futuro obtener mejores resultados.

Con relación a las dependencias administrativas, los docentes exponen que los factores socioeconómicos (materiales, alimentación, nivel de educación, entre otros), afectan o influyen en el desempeño que puedan tener los alumnos en la clase de educación física y así también en los resultados que obtienen en las evaluaciones.

Finalmente los profesores de educación física aprueban la medición SIMCE, pero señalan que varios factores deben mejorar para la obtención de buenos resultados en futuras aplicaciones, partiendo por mejoras desde el mismo sistema de educación, implementando más tiempo a la actividad física dentro de la jornada escolar, entregando mayor importancia a las actividades físicas y deportivas, y realizando modificaciones al SIMCE de educación física, a partir de los resultados obtenidos en la aplicación piloto. 
CAJAS L.B.; BARRAZA G.F.; OLIVARES C.N.; SILVA P.T.; VILLALOBOS G.D. Creencias asociadas a la prueba SIMCE de educación física por parte de los docentes de educación física de colegios particulares, subvencionados y municipales de la comuna de San Felipe. Mot. Hum., 12(2): 21-25, 2011.

\title{
REFERENCIAS BIBLIOGRAFICAS
}

1. Programa de Evaluación del Rendimiento Escolar” (PER) en 1982

2. Schiefelbein, E. (1992). “Análisis del SIMCE y sugerencias para mejorar su impacto en la calidad”. En Gómez (ed), La realidad en cifras. Estadísticas Sociales. Flacso/INE.

3. Encuesta nacional de salud ENS Chile 2009- 2010, Pontificia Universidad Católica de Chile.

4. Métodos Científicos de observación en Educación. Beatriz P. Acuña. 2011. Editorial Visión Libros. Madrid España.

5. Rodríguez, G., Gil, J. y García, E. Metodología de la Investigación Cualitativa. 2ª Ed. Málaga: Aljibe. 1999.

6. El vigía, Resumen Ejecutivo, Encuesta Nacional de Salud, 2003. Supl 8 (20), 2004.

\begin{abstract}
This research presents descriptive case type as a general objective to know the beliefs associated with SIMCE physical education by physical education teachers in private schools, subsidized and municipal schools in the borough of San Felipe. Was carried out in the period from March to December 2011. This qualitative techniques, in-depth interviews or unstructured open-ended questions for physical education teachers in order to know the beliefs associated with SIMCE of Physical Education. Expose content related to the measurement system of education in Chile, administrative offices, rates of physical inactivity, obesity and implications of these, according to studies in school performance and in the teaching-learning process. It is hoped that this research, provide basic and detailed information to substantiate further studies of greater depth and analysis, as well as being the basis for future action projects in the field of educational context specifically in the area of educational assessment policies in the country.
\end{abstract}

Key words: Simce, Teachers, physical, administrative units.

\section{Dirigir Correspondencia a:}

Boris Cajas Luna

Fono: 92538693

Email: bcajas@gmail.com

Agua Santa 7055, sector Rodelillo, Viña del Mar, Chile.

\section{RECIBIDO: 1-12-2011}

ACEPTADO: 29-12-2011 